\title{
REMOCIÓN DE MERCURIO EMPLEANDO CARBÓN ACTIVADO PREPARADO A PARTIR DE AGUAJE (Mauritia flexuosa)
}

\author{
Daniel Obregón-Valencia*, Ilse Acosta ${ }^{\mathrm{a}}$, Isabel Díaz ${ }^{\mathrm{a}}$, Rosario Sun-Kou ${ }^{\mathrm{b}}$
}

\begin{abstract}
RESUMEN
El objetivo de la presente investigación es aplicar el carbón activado obtenido a partir de semillas de aguaje (Mauritia flexuosa) y aplicarlo para la remoción de mercurio. El carbón activado fue modificado con óxido de manganeso para realizar una comparación entre el carbón modificado y sin modificar. El empleo de ultrasonido y en una solución $\mathrm{pH} 9$ favoreció la fijación del manganeso en la superficie del carbón, lo cual fue determinado empleando las técnicas de DRX y la técnica de ICP OES. Los ensayos cinéticos realizados a un $\mathrm{pH}$ 3,5 (encima del potencial de carga cero) con una solución de concentración de 5 ppm de mercurio muestran una mayor capacidad de adsorción para el carbón modificado a $\mathrm{pH}$ 9 ajustándose al modelo de pseudo segundo orden. Las isotermas de adsorción indican un comportamiento predominantemente del tipo Freundlich.
\end{abstract}

Palabras clave: carbón activado, aguaje, mercurio, adsorción.

\section{REMOVAL OF MERCURY USING ACTIVATED CARBON PREPARED FROM AGUAJE (Mauritia flexuosa)}

\begin{abstract}
The objective of the present investigation is apply activated carbon obtained prepared from aguaje seeds (Mauritia flexuosa) for the removal of mercury. Activated carbon obtained was modified with manganese oxide to compare the adsorption capacity between the modified and unmodified carbon. The use of ultrasound in a solution with a $\mathrm{pH} 9$ favored the fixation of manganese on the surface of the activated carbon, which was determined using the techniques of DRX and ICP-OES. The kinetic tests carried out at $\mathrm{pH} \mathrm{3,5} \mathrm{(over} \mathrm{zero} \mathrm{charge} \mathrm{potential)}$ with a concentration of 5 ppm of mercury show a higher adsorption capacity at modified carbon at $\mathrm{pH} 9$ in accordance with the pseudo second order model. Adsorption isotherms indicate a predominantly Freundlich behavior.
\end{abstract}

Key words: activated carbon, aguaje, mercury, adsorption.

\footnotetext{
a Instituto de Corrosión y Protección, Pontificia Universidad Católica del Perú. Av. Universitaria 1801, Lima 32, Perú. Email: daniel.obregon@pucp.edu.pe*

b Sección Química, Departamento Académico de Ciencias, Pontificia Universidad Católica del Perú. Av.

Universitaria 1801, Lima 32, Perú. Email: msun@pucp.edu.pe
} 


\section{INTRODUCCIÓN}

El mercurio es considerado uno de los elementos con mayor presencia y toxicidad en el ambiente, junto con el plomo y el arsénico. El mercurio no es biodegradable y puede ser transportado a través de la cadena alimenticia.

Las principales fuentes de contaminación por mercurio en el medio acuático son las industrias de cloro-álcali, pinturas, papel, refinación de petróleo, procesamiento de caucho y fertilizantes, principalmente.

Estudios recientes en los que se ha utilizado óxidos metálicos de manganeso, hierro, titanio, aluminio y cerio han mostrado una adsorción o retención significativa y selectiva hacia muchos iones metálicos tóxicos, constituyéndose así en adsorbentes prometedores para la remoción de metales de sistemas acuosos ${ }^{1}$. Sin embargo, debido a esto, los óxidos metálicos se aglomeran y su alta capacidad de selectividad se reduce o se pierde. A pesar de que los óxidos de manganeso son considerados unos importantes removedores de trazas metálicas de diversos metales en medios acuosos ${ }^{2}$ hay muy poca información acerca de la remoción de mercurio. Para mejorar el uso de los óxidos metálicos en tratamientos de aguas residuales se investigan métodos en los cuales estos compuestos se impregnan en soportes con alta porosidad, estabilidad térmica y frente a medios ácidos y básicos ${ }^{3}$. Por tal motivo, en la literatura se encuentra la preparación de diversos compósitos de grafeno y óxidos metálicos empleados en la remoción de metales ${ }^{4}$, sin embargo, dichos métodos de síntesis son poco reproducibles a gran escala.

Estudios previos han demostrado que el carbón activado preparado a partir de semillas de aguaje (Mauritia flexuosa) tiene una alta capacidad de remoción de metales pesados ${ }^{5,6}$. La modificación del carbón activado con nitrato de manganeso mediante una impregnación húmeda para adsorber cobre y plomo revela que la adsorción se incrementó hasta en un $30 \%$, en el caso del plomo la capacidad de adsorción se incrementó de 2,94 a 3,31 mg/g y para el cobre de 0,68 a $0,91 \mathrm{mg} / \mathrm{g}^{7}$.

\section{PARTE EXPERIMENTAL}

\section{Materiales y métodos}

\section{Materia prima}

Las semillas de aguaje fueron obtenidas de la ciudad de Pucallpa, provincia de Ucayali, Perú. Fue eliminado cualquier residuo orgánico de las semillas, las cuales fueron secadas a $80^{\circ} \mathrm{C}$ y luego partidas hasta obtener un tamaño de particular menor que $10 \mathrm{~mm}$.

\section{Preparación y modificación del carbón activado}

El carbón activado seleccionado fue preparado de acuerdo al método presentado en un trabajo previo $^{5}$. Se seleccionó el carbón activado AG0.75_600, que significa que fue preparado 
con un grado de impregnación de $0.75 \mathrm{~g}_{\mathrm{H} 3 \mathrm{PO} 4} / \mathrm{g}_{\text {PRECURSOR }} \mathrm{y}$ fue activado a $600{ }^{\circ} \mathrm{C}$. El carbón activado empleado para la modificación fue el que presentó una mayor remoción de $\mathrm{Cd}, \mathrm{Cr}$ y $\mathrm{Pb}^{5,6}$. La muestra AG0.75_600 en adelante será denominada muestra sin modificar SMOD. Posteriormente, SMOD fue modificada con nitrato de manganeso.

La modificación con nitrato de manganeso se realizó mediante una impregnación húmeda. Se prepararon dos soluciones de manganeso a partir de $\mathrm{Mn}\left(\mathrm{NO}_{3}\right)_{2} \cdot 4 \mathrm{H}_{2} \mathrm{O}$ (Merck, grado P.A.) a un $\mathrm{pH}$ de 3 y 9 aproximadamente. Se colocó $20 \mathrm{~mL}$ de la solución preparada previamente a una concentración establecida y se mezcló con $80 \mathrm{mg}$ de CA (carbón activado). La mezcla se realizó bajo un baño de ultrasonido. Luego, las mezclas fueron colocadas bajo agitación constante a $40{ }^{\circ} \mathrm{C}$ por 1 hora. Las mezclas se dejaron enfriar y luego dejadas en agitación durante toda una noche. Después de un proceso de filtración el CA se secó a $60{ }^{\circ} \mathrm{C}$ en una estufa durante toda una noche. Las muestras secas se colocaron dentro de un reactor tubular en una atmósfera inerte y la temperatura fue llevada a $250^{\circ} \mathrm{C}$ a una velocidad de $5^{\circ} \mathrm{C} / \mathrm{min}$. El CA modificado a pH 3 y pH 9 serán denominados MOD_pH3 y MOD_pH9, respectivamente.

\section{Caracterización fisicoquímica}

\section{Ensayos de adsorción-desorción de $\mathrm{N}_{2}$}

Las propiedades texturales del carbón activado fueron caracterizadas por la técnica de adsorción-desorción de $\mathrm{N}_{2}$ empleando un equipo Micromeritics GEMINI VII, modelo 2390. Se cuantificó la cantidad de $\mathrm{N}_{2}$ gaseoso retenida en la superficie del carbón activado a una temperatura de $77 \mathrm{~K}$ hasta alcanzar una presión relativa de 1. El área superficial total fue calculada de acuerdo al método BET. El área microporosa fue calculada por el método t-plot. El área mesoporosa fue obtenida por la diferencia entre el área superficial total y el área microporosa.

\section{Titulación Boehm}

La determinación de los grupos funcionales ácidos de la superficie del CA fue realizada por el método de titulación BOEHM. Se realizó la preparación de tres soluciones $0,05 \mathrm{M}$ de $\mathrm{NaOH}$ (Scharlau, ACS), $\mathrm{NaHCO}_{3}$ (J.T. Backer, ACS) y $\mathrm{Na}_{2} \mathrm{CO}_{3}$ (Fisher Chemical, ACS), respectivamente. Se pesó $125 \mathrm{mg}$ de cada CA y se mezclaron por 24_horas bajo agitación constante con $20 \mathrm{~mL}$ de cada una de las soluciones preparadas anteriormente. Se filtró y las soluciones remanentes fueron tituladas con 0,05 M de_HCl (Merck, P.A.) estandarizado previamente de acuerdo al método ASTM E200-08.

\section{Determinación del punto de carga cero}

Se determinó el punto de carga cero de la superficie preparando soluciones de diversos $\mathrm{pH}$ en un rango de 2 a 8. Se mezcló $50 \mathrm{mg}$ de CA con $20 \mathrm{~mL}$ de las soluciones preparadas previamente y se mantuvo bajo agitación constante por 24_horas. Luego, la mezcla se filtró y se determinó su $\mathrm{pH}$ empleando un potenciómetro marca $\overline{\mathrm{W}} \mathrm{TW}$, modelo 537 y un electrodo de $\mathrm{pH}$ de $\mathrm{Ag} / \mathrm{AgCl}$ con electrolito líquido de $\mathrm{KCl}$ 0,1 M marca SenTix, modelo 81. Se generó una gráfica de los datos del $\mathrm{pH}$ inicial y $\mathrm{pH}$ final de las soluciones, la curva obtenida corta el eje X, siendo el punto de corte sobre el eje X el punto de carga cero. 


\section{Difracción de rayos $\mathrm{X}$}

La determinación de los óxidos formados en la superficie del CA se realizó mediante el análisis de rayos $\mathrm{X}$ de los CA modificados en un difractómetro marca Bruker, modelo D8Focus, con una fuente de radiación $\mathrm{CuK} \alpha \mathrm{y}$ una longitud de onda de radiación de rayos $\mathrm{X}$ de $1,5406 \AA$.

\section{Ensayos de análisis elemental}

Se realizó la determinación del contenido de manganeso presente en los CA modificados mediante un tratamiento de digestión ácida de los mismos y su posterior análisis en un equipo ICP-OES marca Perkin Elmer, modelo Optima 3000DV.

\section{Microscopía electrónica de barrido}

Se realizó un análisis SEM con un equipo FEI modelo QUANTA 650 y el análisis EDS por medio de una plataforma INCA 250 EDS con un detector XMax de $20 \mathrm{~mm}$ acoplado al equipo.

\section{Experimentos de adsorción}

Todas las soluciones de mercurio fueron preparadas a partir de un estándar de mercurio de 1000 mg/L (Merck, CertiPur). Para la determinación de mercurio se empleó la técnica de espectroscopia de absorción atómica mediante la generación de vapor frío (AAS-CV) basado en el método EPA 245.1.

\section{Ensayos cinéticos}

Se empleó una solución estándar de mercurio de 1000 mg/L (Merck, grado Certipur). Para los ensayos de cinética de adsorción se empleó $20 \mathrm{~mL}$ de una solución de 5 ppm de mercurio y $12 \mathrm{mg}$ de CA a temperatura ambiente y un $\mathrm{pH}$ de 2 y 3,5. Se mantuvieron en agitación constante por un tiempo determinado (entre 5 y $1400 \mathrm{~min}$ ).

A fin de entender los mecanismos de adsorción, los resultados experimentales se ajustaron a los modelos de pseudo primer orden, pseudo segundo orden y difusión intraparticular que se presentan en la tabla 1.

\section{Ensayos de isotermas}

Los ensayos para las isotermas se realizaron empleando un tiempo de contacto de 4 horas en un rango de concentraciones de 10 a 40 ppm para un pH de 2 y de 10 a $70 \_p p m$ para un pH de 3,5 .

Los resultados experimentales se ajustaron a los modelos de Langmuir, Freundlich, RedlichPeterson y Temkin que se presentan en la tabla 2. 
Tabla 1. Modelos cinéticos de adsorción.

\begin{tabular}{|c|c|}
\hline Representación matemática & Parámetros \\
\hline \multicolumn{2}{|l|}{ Pseudo primer orden } \\
\hline $\begin{array}{l}\log \left(q_{e}-q_{t}\right)= \\
\log \left(q_{e}\right)-\left(\frac{k_{1}}{2,303}\right) t\end{array}$ & $\begin{array}{l}\mathrm{k}_{1}(1 / \mathrm{min})=\text { velocidad inicial de adsorción } \\
\mathrm{q}_{\mathrm{e}}(\mathrm{mg} / \mathrm{g})=\text { capacidad de adsorción en el } \\
\text { equilibrio } \\
\mathrm{q}_{\mathrm{t}}(\mathrm{mg} / \mathrm{g})=\text { capacidad de adsorción al tiempo " } \mathrm{t} \text { " }\end{array}$ \\
\hline \multicolumn{2}{|l|}{ Pseudo segundo orden } \\
\hline$\frac{\mathrm{t}}{\mathrm{q}_{\mathrm{t}}}=\frac{1}{\mathrm{k}_{2} \mathrm{q}_{\mathrm{e}}^{2}}+\frac{1}{q_{e}} t$ & $\begin{array}{l}\mathrm{k}_{2}(\mathrm{~g} / \mathrm{mg}-\mathrm{min})=\text { velocidad inicial de adsorción } \\
\mathrm{q}_{\mathrm{e}}(\mathrm{mg} / \mathrm{g})=\text { capacidad de adsorción en el } \\
\text { equilibrio } \\
\mathrm{q}_{\mathrm{t}}(\mathrm{mg} / \mathrm{g})=\text { capacidad de adsorción en el tiempo } \\
\text { " } \mathrm{t} \text { " }\end{array}$ \\
\hline \multicolumn{2}{|l|}{ Difusión intraparticular } \\
\hline$q_{t}=k_{\text {diff }} t^{0,5}+C$ & $\begin{array}{l}\mathrm{k}_{\text {diff }}\left(\mathrm{mg} / \mathrm{g}-\mathrm{min}^{1 / 2}\right)=\text { constante de difusión } \\
\text { intraparticular } \\
\mathrm{C}(\mathrm{mg} / \mathrm{g})=\text { relacionado con el espesor de la capa } \\
\text { limitante }\end{array}$ \\
\hline
\end{tabular}

La desviación estándar normalizada entre los valores experimentales y los valores predichos por los modelos fue empleada para evaluar el modelo que presentaba el mejor ajuste. La desviación estándar normalizada fue calculada de la siguiente manera:

$$
\Delta \text { qe }(\%)=100 \sqrt{\frac{\sum[q e(e x p)-q e(p r o) / q e(\exp )]^{2}}{N}}
$$

donde, qe(exp) es el valor experimental, qe(pro) es el valor correspondiente al qe pronosticado de acuerdo a la ecuación bajo estudio, $\mathrm{N}$ es el número de mediciones. En este estudio el menor valor obtenido de desviación estándar normalizado indicó el mejor ajuste de los resultados experimentales. 
Tabla 2. Modelos de isotermas de adsorción.

\begin{tabular}{ll}
\hline \multicolumn{1}{c}{ Representación matemática } & \multicolumn{1}{c}{ Parámetros } \\
\hline Freundlich & $q_{e}(\mathrm{mg} / \mathrm{g})=$ capacidad de adsorción en el \\
& equilibrio \\
& $C_{e}(\mathrm{mg} / \mathrm{L})=$ Concentración del soluto en la \\
solución en el equilibrio
\end{tabular}




\section{RESULTADOS Y DISCUSIÓN}

\section{Caracterización fisicoquímica}

\section{Ensayos de adsorción-desorción de $\mathbf{N}_{2}$}

En el análisis textural mediante la técnica BET se observa que las muestras presentan una isoterma de adsorción de $\mathrm{N}_{2}$ del Tipo I correspondiente a materiales microporosos (figura 1).

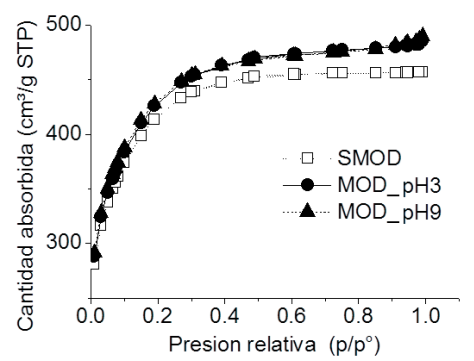

Figura 1. Isotermas de adsorción-desorción de $\mathrm{N}_{2}$ de los CA.

Se puede observar que el área superficial total y tamaño de poro se incrementa en el caso de los CA modificados pero no de manera significativa, siendo mayor para el caso de MOD_ pH9 (tabla 3).

Tabla 3. Análisis texturales del CA sin modificar y modificado.

\begin{tabular}{ccccc}
\hline \multirow{2}{*}{ Muestra } & \multicolumn{3}{c}{ Área $\left(\mathrm{m}^{2} / \mathrm{g}\right)$} & Tamaño de poro \\
& Total & Microporosa & Mesoporosa & $(\mathrm{nm})$ \\
\hline SMOD & 1484,42 & 1353,04 & 131,38 & 1,08 \\
MOD_pH 3 & 1529,66 & 1388,03 & 141,63 & 1,12 \\
MOD_pH9 & 1537,36 & 1384,19 & 153,17 & 1,14
\end{tabular}

\section{Titulación Boehm}

La acidez superficial total de los CA fue muy similar entre ellos (tabla 4). En los CA modificados se observa una disminución de los grupos carboxílicos y un incremento de los grupos fenólicos respecto a SMOD. La disminución de los grupos carboxílicos y lactónicos y el aumento de los grupos fenólicos confieren un carácter básico a la superficie del carbón activado. Los valores de titulación Boehm son referenciales y sirven para la comparación entre los CA preparados. 
Tabla 4. Resultados de acidez superficial de acuerdo a la titulación Boehm.

\begin{tabular}{ccccc}
\hline Muestra & $\begin{array}{c}\text { Acidez superficial } \\
\text { total }\left(\mathrm{H}^{+} \mathrm{mmol} / \mathrm{g}\right)\end{array}$ & Fenólicos & $\begin{array}{c}\text { Grupos } \\
\text { Lactónicos }\end{array}$ & Carboxílicos \\
\hline SMOD & 0,9714 & 0,2043 & 0,384 & 0,3832 \\
MOD_pH3 & 1,0166 & 0,3389 & 0,337 & 0,3403 \\
MOD_pH9 & 0,9328 & 0,5087 & 0,084 & 0,3405
\end{tabular}

\section{Determinación del punto de carga cero}

Como se indicó anteriormente, el punto de carga cero se determinó a partir de las curvas generadas con los $\mathrm{pH}$ iniciales y finales de cada solución que estuvo en contacto con el CA. Las curvas obtenidas se presentan a continuación:

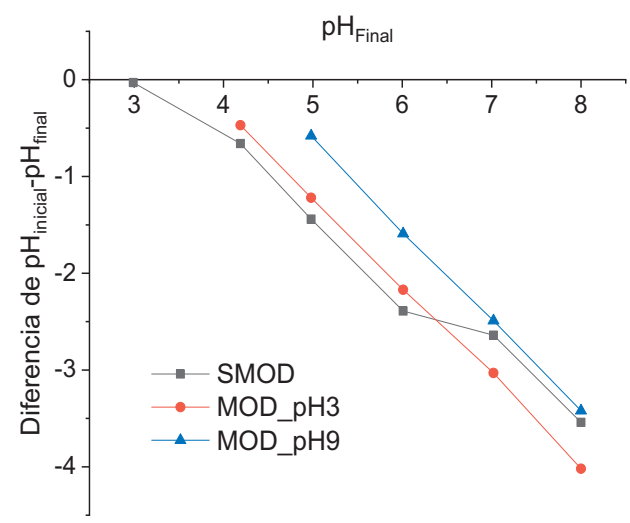

Figura 2. DRX de los CA modificados con manganeso.

Las ecuaciones de las curvas obtenidas a partir de dichos puntos se presentan a continuación:

SMOD: $y=-0,7049 x+2,1158$

MOD_pH3: $\quad y=-0,9221 x+3,3875$

MOD_pH9: $\quad y=-0,9355 x+4,0632$

El valor de potencial de carga cero se obtiene al igualar a cero el valor de "y". El punto de carga cero se incrementó en los CA modificados, alcanzando el máximo en MOD_pH9 (tabla 5). Esto indica que los CA modificados presentan una superficie cargada negativamente a $\mathrm{pH}$ mayores; de esta manera se ve favorecida la adsorción de metales al aumentar el $\mathrm{pH}$ de la solución. Esto se puede atribuir al incremento del carácter básico de la superficie del carbón activado debido al incremento de los grupos fenólicos con respecto a los otros grupos funcionales presentes en su superficie ${ }^{8}$. 
Tabla 5. Punto de carga cero de la superficie de los CA.

\begin{tabular}{cccc}
\hline Muestra & SMOD & $\mathrm{MOD}_{-}$pH3 & MOD _pH9 \\
& & & \\
\hline pHpzc & 3,00 & 3,67 & 4,35 \\
& & & \\
\hline
\end{tabular}

Lalhmunsiama et al. ${ }^{7}$ reportaron la adsorción de $\mathrm{Cu}^{2+} \mathrm{y} \mathrm{Pb}^{2+}$ sobre carbón activado modificado con óxidos de manganeso cuya carga cero de superficie fue 6,2. Se determinó que la adsorción de $\mathrm{Cu}^{2+} \mathrm{y} \mathrm{Pb}^{2+}$ es menor a $\mathrm{pH}$ más ácidos. Se ha reportado en zeolitas modificadas ${ }^{9}$ con manganeso un pHpzc de 3,8 y en arena modificada ${ }^{10}$ con manganeso un valor de 6,28. Se ha reportado que la presencia de óxidos de manganeso mejora las propiedades redox e incrementa la cantidad de oxígeno adsorbido en la superficie de materiales ${ }^{11}$ como el $\mathrm{CeO}_{2}$ $\mathrm{ZrO}_{2}$. Los óxidos de manganeso sin soporte no presentan estas características debido a su mayor tamaño de partícula ${ }^{12}$. Faulconer y Mazyck ${ }^{13}$ indican que tanto los grupos funcionales oxigenados como el punto de carga zero son factores determinantes en la adsorción de $\mathrm{Hg}$ (II).

\section{Difracción de rayos $\mathrm{X}$}

Se observa en la figura 3, el análisis de DRX de las muestras de CA, la predominancia de dos picos anchos en $2 \theta$ entre $20^{\circ}$ y $30^{\circ}$ y esto se debe a la estructura amorfa del carbón activado ${ }^{14}$. Se puede observar débiles picos de difracción a $2 \theta$ a $32^{\circ}, 36^{\circ} 58^{\circ}$ y $59^{\circ}$ en la muestra modificada en medio básico que corresponden a los cristales de $\mathrm{Mn3O}$. La falta de picos en el carbón modificado en medio ácido puede deberse al menor contenido de manganeso impregnado sobre la superficie del carbón, lo cual fue confirmado con los datos obtenidos por ICP OES sobre el contenido de Mn en los carbones modificados. Los resultados son similares a los obtenidos por Tang, Q. et al. ${ }^{15}$.

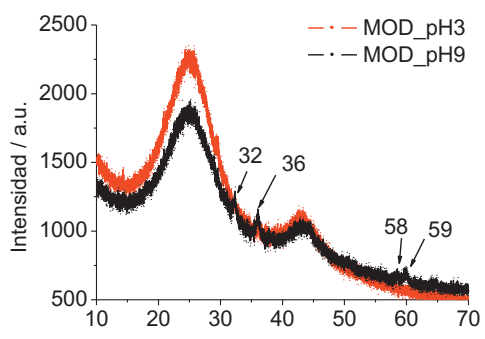

Figura 3. DRX de los CA modificados con manganeso.

Se ha reportado en arenas modificadas ${ }^{16}$ con manganeso la presencia principalmente de vernadita $\left(\delta-\mathrm{MnO}_{2}\right)$ y mediante el empleo de espectroscopía de fotoelectrones emitidos por rayos $\mathrm{X}$ se ha determinado una valencia mixta de $\mathrm{Mn}^{+3} \mathrm{y} \mathrm{Mn}^{+4}$.

\section{Ensayos de análisis elemental}

Los análisis elementales por ICP-OES han determinado un incremento en el contenido de manganeso en los carbones modificados a pH 9 (tabla 6). 
Tabla 6. Determinación del contenido en \% de manganeso presente en los CA.

\begin{tabular}{ccc}
\hline Muestra & MOD_pH3 & MOD_pH9 \\
\hline \% de manganeso & 0,96 & 1,32 \\
\hline
\end{tabular}

\section{Microscopía electrónica de barrido}

Las imágenes de microscopía electrónica de barrido se presentan en la figura 4.
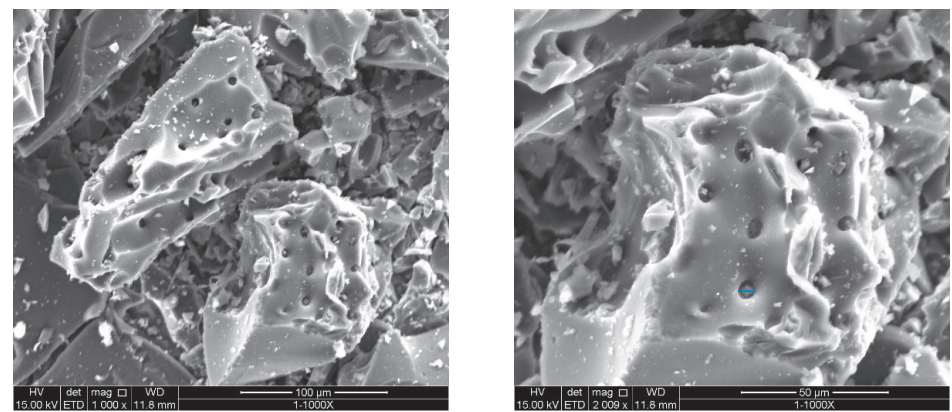

A) SEM de muestra SMOD con aumentos de 1000x y 2009x.
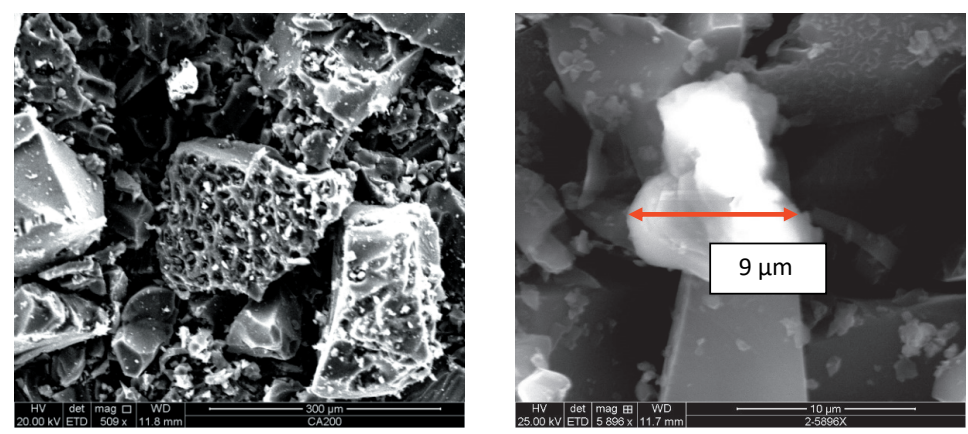

B) SEM de la muestra MOD_pH3 con aumentos de 500X y 5896X.
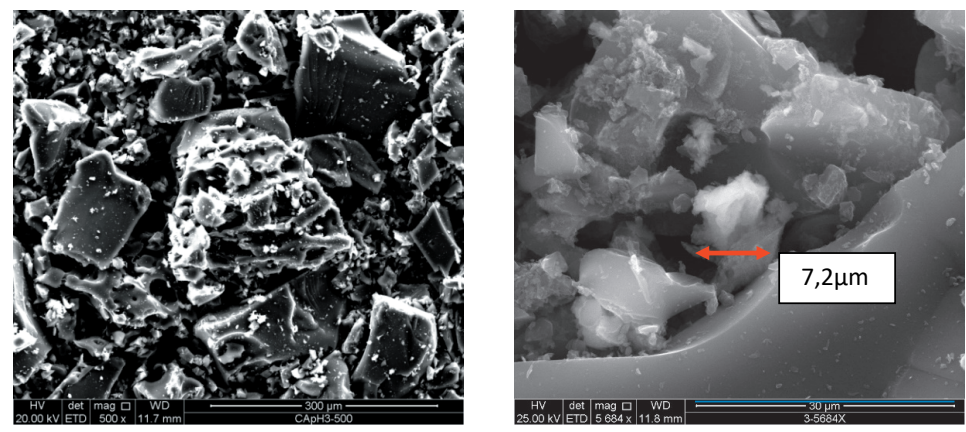

C) SEM de muestra MOD_pH9 con aumentos de 500x y 5684x.

Figura 4. SEM del CA sin modificar (A) y modificado a pH3 (B) y pH 9 (C). 
En la figura 4 - A se presenta la microfotografía obtenida de la muestra SMOD. La figura_4 - A y B presenta la microfotografía obtenida de la muestra MOD_pH3 y MOD_pH9. Se puede observar que la muestra SMOD presenta porosidad muy desarrollada con un tamaño promedio de poro de $8 \mu \mathrm{m}$. Del otro lado, las muestras MOD_pH3 y MOD_pH9 (figura 4 - B y C) muestran una superficie con presencia de óxidos de manganeso. Como se puede observar en la tabla 3 el área superficial total aumenta ligeramente al igual que su área mesoporosa y que el tamaño de poro. Los ligeros cambios en la morfología del material se pueden atribuir al tratamiento poco severo realizado para la modificación con óxido de manganeso del CA.

\section{Ensayos cinéticos e isotermas}

La composición del $\mathrm{Hg}(\mathrm{II})$ en soluciones acuosas es complejo y varía de acuerdo al $\mathrm{pH}$ presentándose como especie dominante a un $\mathrm{pH}<3,0$. El compuesto $\mathrm{Hg}(\mathrm{OH})_{2}$ se vuelve dominante a un $\mathrm{pH}>5,0$. En el rango de $\mathrm{pH}$ de 3 a 5 podemos encontrar las especies ${ }^{9} \mathrm{Hg}(\mathrm{II})$, $\mathrm{HgOH}^{+}$y $\mathrm{Hg}(\mathrm{OH}) 2$.

\section{Ensayos cinéticos}

Los parámetros obtenidos para los modelos cinéticos se presentan en la tabla 7. Los resultados se ajustaron al modelo de pseudo segundo orden, lo cual indicaría que la etapa limitante es el proceso de sorción en vez del de difusión. La capacidad de adsorción se incrementó con el aumento del pH de la solución de 2 a 3,5. La menor capacidad de adsorción en una solución de $\mathrm{pH}_{2} 2$ se atribuye a la competencia entre los iones $\mathrm{H}+$ contra los $\mathrm{Hg}$ (II) por el mismo sitio activo del adsorbente.

Tabla 7. Parámetros cinéticos.

\begin{tabular}{ccccccc}
\hline \multirow{2}{*}{ Muestra } & \multicolumn{3}{c}{ Pseudo primer orden } & \multicolumn{3}{c}{ Pseudo segundo orden } \\
& $\mathrm{q}_{\mathrm{e}}$ & $\mathrm{k}_{1}$ & $\mathrm{R}^{2}$ & $\mathrm{q}_{\mathrm{e}}$ & $\mathrm{k}_{2}$ & $\mathrm{R}^{2}$ \\
\hline Solución pH 2 & & & & & & \\
SMOD & 3,178 & 0,008 & 0,631 & 7,71 & 0,006 & 1,00 \\
MOD_pH3 & - & - & - & 7,61 & 0,021 & 1,00 \\
MOD_pH9 & 3,004 & 0,007 & 0,809 & 7,57 & 0,014 & 1,00 \\
Solución pH 3,5 & & & & & & \\
SMOD & 8,98 & 0,042 & 0,76 & 8,07 & 0,010 & 1,00 \\
MOD_pH3 & 5,82 & 0,030 & 0,96 & 7,72 & 0,012 & 1,00 \\
MOD_pH9 & 8,93 & 0,041 & 0,79 & 8,21 & 0,010 & 1,00 \\
\hline
\end{tabular}

Leyenda: Unidades $\mathrm{q}_{\mathrm{e}}=\mathrm{mg} \mathrm{g}^{-1} \mathrm{y} \mathrm{K}_{1}=\min ^{-1}, \mathrm{~K}_{2}=\mathrm{g} \mathrm{mg}^{-1} \min ^{-1}$.

La adsorción de mercurio está relacionada principalmente a la captura de sus especies catiónicas, y en menor medida de sus especies aniónicas que se encuentran en pH básicos. Las especies $\mathrm{HgOH}^{+}$y $\mathrm{Hg}$ (II) son capturadas en mayor medida que las $\mathrm{HgCl}^{+} .{ }^{15} \mathrm{~A}_{\text {condiciones }}$ ácidas ( $\mathrm{pH}$ 3-5), el efecto del $\mathrm{pH}$ en la remoción de $\mathrm{Hg}$ (II) se da mediante repulsión 
electrostática debido a que se espera que el adsorbente y adsorbato se encuentran cargados positivamente. Sin embargo, al disminuir la concentración de $\mathrm{H}^{+}$, manteniendo constante la concentración de $\mathrm{Hg}^{2+}$, produce un incremento en su remoción debido a la disminución de la competencia con los iones $\mathrm{H}^{+}$por los sitios de sorción y al cambio de la carga de la superficie del CA al pasar su punto de carga cero ${ }^{15,16,17,18}$.

La muestra SMOD presenta una mayor capacidad de adsorción a pH 2 siendo superada por la muestra MOD_pH9 al incrementarse el pH de la solución a 3,5. Se puede observar un incremento de los grupos fenólicos en MOD_pH9, además, que presenta un mayor número de sitios activos debido a la presencia de los óxidos de manganeso. Se ha reportado que existe una fuerte interacción del mercurio con los grupos $\mathrm{MnO}_{2} \mathrm{o} \mathrm{MnOOH}$.

La formación de $\mathrm{MnO}_{2}=\mathrm{Hg}(\mathrm{II})$ o $\mathrm{MnOOH}=\mathrm{Hg}(\mathrm{II})$ puede ocurrir durante el proceso de sorción $^{19,20}$ por medio de un intercambio de ligandos o la formación de complejos en la superficie del carbón activado modificado con óxido de manganeso ${ }^{20}$. El incremento del $\mathrm{pH}$ disminuye el efecto competitivo de los iones $\mathrm{H}^{+}$. Además, la superficie del carbón activado se carga negativamente al estar sobre su punto de carga cero. Se puede observar que la muestra SMOD tiene un pHPZC de 3 y la muestra MOD_pH9 de 4,75 (tabla_5), lo cual estaría favoreciendo la adsorción en la muestra SMOD. La capacidad de adsorción en los ensayos de cinética fue de 8,00 y 8,14 mg/g para el SMOD y MOD_pH9, lo cual está acorde con los resultados cinéticos del modelo de pseudo segundo orden (tabla_7). Además, si bien la diferencia es muy pequeña, el método de determinación de $\mathrm{Hg}$ alcanza límites de detección de $0,1 \mu \mathrm{g} / \mathrm{L}$ de mercurio con un valor de incertidumbre estimada de $0,3 \mu \mathrm{g} / \mathrm{L}$. El Hg(II) presenta una toxicidad alta a muy pequeñas concentraciones, por tal motivo, es de suma importancia la remoción total del mercurio, asimismo, las pequeñas diferencias en las concentraciones presentes en el agua tienen una importancia indiscutible.

De acuerdo al modelo de pseudo segundo orden, la velocidad de adsorción es determinada por las reacciones químicas. Al inicio, el proceso de adsorción es rápido debido a la mayor cantidad de sitios activos presentes en la superficie del CA que pueden combinarse con el $\mathrm{Hg}(\mathrm{II})$.

La velocidad de adsorción disminuye con el incremento del tiempo de adsorción debido a la reducción de sitios activos sobre la superficie del CA y la disminución del gradiente de concentración entre la interfaz sólido-líquido (figura 5). 

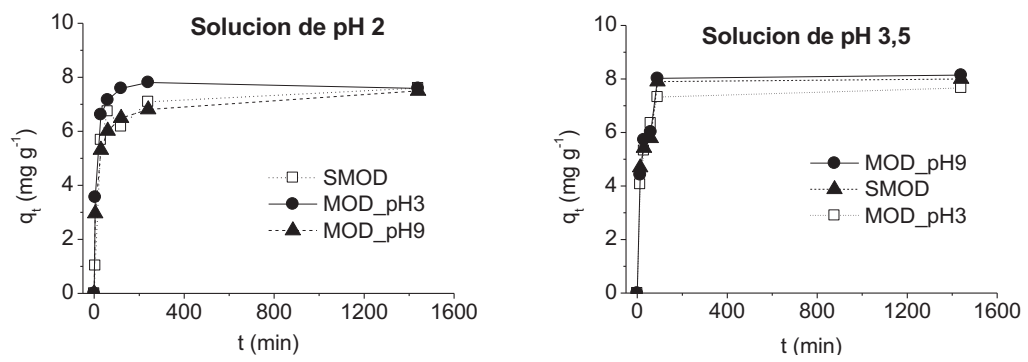

Figura 5. Curvas cinéticas de adsorción de mercurio en las muestras seleccionadas en una solución de $\mathrm{pH} 2$ y 3,5 .

Arshadi ${ }^{1}$ y Lu et al..$^{21}$ encontraron que el mejor ajuste al modelo de pseudo segundo orden. Lu et al..$^{21}$ mostraron que el modelo cinético de adsorción de pseudo segundo orden presentó un mejor ajuste, sugiriendo que el proceso de adsorción ocurre en dos etapas; una primera etapa consistiría en una rápida propagación del $\mathrm{Hg}$ (II) sobre la superficie del carbón activado, seguido de una etapa lenta en la cual el $\mathrm{Hg}$ (II) pasa al interior de los microporos y se combina con los sitios activos internos. Además, reportaron que el área superficial específica no tuvo mayor influencia en la adsorción del mercurio.

\section{Modelo de difusión intraparticular}

No se observó una relación lineal entre las variables qt y $\mathrm{t}^{1 / 2}$; sin embargo, se pudo diferenciar tres etapas diferentes.

En la primera etapa se observa una velocidad de adsorción alta, en esta etapa solo existe la resistencia externa de transferencia de masas del CA. En la segunda etapa disminuye la velocidad de adsorción, sin embargo, no se observa intercepción con el origen indicando que la difusión intraparticular no es el factor limitante (figura 6). En la última etapa se alcanza el equilibrio y la difusión intraparticular disminuye drásticamente debido a la extremadamente baja concentración del analito remanente en la solución (tabla 8).

Tabla 8. Parámetros de difusión intraparticular a distintos $\mathrm{pH}$.

\begin{tabular}{cccccccccc}
\hline Muestra & $\mathrm{k}_{\text {dif1 }}$ & $\mathrm{k}_{\text {dif2 }}$ & $\mathrm{k}_{\text {dif3 }}$ & $\mathrm{C}_{1}$ & $\mathrm{C}_{2}$ & $\mathrm{C}_{3}$ & $\mathrm{R}_{1}{ }^{2}$ & $\mathrm{R}_{2}{ }^{2}$ & $\mathrm{R}_{3}{ }^{2}$ \\
\hline Solución pH 2 & & & & & & & & & \\
SMOD & 0,46 & 0,58 & 0,04 & 0,00 & 1,05 & 6,03 & 1,00 & 0,67 & 0,75 \\
MOD_pH3 & 1,59 & 0,45 & 0,00 & 0,00 & 3,27 & 7,73 & 1,00 & 0,82 & 0,13 \\
MOD_pH9 & 1,32 & 0,40 & 0,04 & 0,00 & 2,55 & 6,18 & 1,00 & 0,88 & 0,98 \\
Solución pH 3.5 & & & & & & & & & \\
SMOD & 1,30 & 0,26 & 0,04 & 0,00 & 3,84 & 6,45 & 1,00 & 0,94 & 0,33 \\
MOD_pH3 & 1,13 & 0,55 & 0,03 & 0,00 & 2,17 & 6,57 & 1,00 & 0,99 & 0,54 \\
MOD_pH9 & 1,23 & 0,38 & 0,04 & 0,00 & 3,27 & 6,64 & 1,00 & 0,84 & 0,34 \\
\hline
\end{tabular}

Leyenda: Unidades de $\mathrm{K}_{\mathrm{dif} 1}, \mathrm{~K}_{\mathrm{dif} 2}, \mathrm{~K}_{\mathrm{dif}}=\mathrm{mg} \mathrm{g}^{-1} \min ^{-1 / 2}$. 

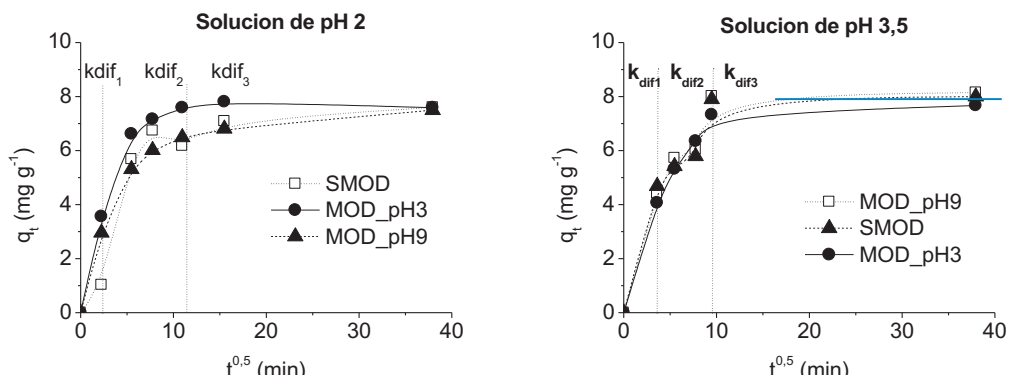

Figura 6. Cinéticas de adsorción de las muestras seleccionadas de acuerdo con el modelo de difusión intraparticular en una solución de pH 2 y 3,5.

\section{Isotermas de adsorción}

Los ensayos de isotermas en una solución a pH 2 dieron una máxima capacidad de adsorción para SMOD, siendo similares los resultados para los carbones modificados (tabla 9). El modelo de Freundlich presentó un coeficiente de correlación alto y una menor desviación estándar normalizada. Por tal motivo, puede establecer que el proceso de adsorción de mercurio se llevó a cabo en sitios activos heterogéneos y con la formación de multicapas.

Los ensayos de isotermas en una solución a pH 3,5 (figura 7) dieron como resultado una capacidad de adsorción similar para las muestras SMOD y MOD_pH9. Se evidencia un proceso de adsorción con tendencia al tipo Freundlich. Estos resultados son comparables con los obtenidos por Rao et al..$^{22}$. 
Tabla 9. Resultados experimentales de las isotermas de adsorción.

\begin{tabular}{|c|c|c|c|c|c|c|}
\hline \multirow{2}{*}{$\begin{array}{c}\text { Medio } \\
\text { Muestra }\end{array}$} & \multicolumn{3}{|c|}{ Solución pH 2} & \multicolumn{3}{|c|}{ Solución pH 3.5} \\
\hline & SMOD & MOD_pH3 & MOD_pH9 & SMOD & MOD_pH3 & MOD_pH9 \\
\hline \multicolumn{7}{|c|}{ Freundlich } \\
\hline $\mathbf{K}_{\mathbf{F}}$ & 15,65 & 12,83 & 12,59 & 23,15 & 19,87 & 23,98 \\
\hline $1 / n$ & 0,23 & 0,24 & 0,23 & 0,36 & 0,41 & 0,33 \\
\hline $\mathbf{r}^{2}$ & 1,00 & 1,00 & 1,00 & 0,96 & 0,93 & 0,96 \\
\hline$\% \Delta \mathbf{E}$ & 3,17 & 1,68 & 1,91 & 27,09 & 22,47 & 20,52 \\
\hline \multicolumn{7}{|c|}{ Langmuir } \\
\hline qm & 33,62 & 29,39 & 27,79 & 79,66 & 76,52 & 78,29 \\
\hline $\mathrm{K}_{\mathrm{L}}$ & 0,56 & 0,43 & 0,46 & 0,33 & 0,27 & 0,27 \\
\hline $\mathrm{r}^{2}$ & 0,992 & 0,987 & 0,994 & 0,998 & 0,976 & 0,973 \\
\hline$\% \Delta \mathrm{E}$ & 18,71 & 17,52 & 14,91 & 13,02 & 26,13 & 33,83 \\
\hline \multicolumn{7}{|l|}{ Temkin } \\
\hline B & 0,48 & 0,54 & 0,57 & 0,17 & 0,16 & 0,20 \\
\hline $\mathbf{A}_{\mathbf{T}}$ & 20,73 & 15,11 & 16,56 & 4,84 & 3,93 & 8,35 \\
\hline $\mathbf{r}^{2}$ & 0,97 & 0,98 & 0,98 & 0,92 & 0,75 & 0,77 \\
\hline$\% \Delta \mathbf{E}$ & 5,71 & 4,71 & 4,42 & 19,08 & 26,74 & 30,64 \\
\hline \multicolumn{7}{|c|}{ Redlich Peterson } \\
\hline G & 0,79 & 0,78 & 0,78 & 0,78 & 0,82 & 1,00 \\
\hline$A_{R P}$ & 265 & 200 & 270 & 42 & 42 & 79 \\
\hline $\mathrm{B}_{\mathrm{RP}}$ & 15,89 & 14,68 & 20,58 & 1,03 & 1,05 & 1,37 \\
\hline$r^{2}$ & 1,00 & 1,00 & 1,00 & 0,93 & 0,94 & 0,94 \\
\hline$\% \Delta \mathrm{E}$ & 4,24 & 2,04 & 2,08 & 26,60 & 29,24 & 217,23 \\
\hline \multicolumn{7}{|c|}{ Dubinin-Radushkevich } \\
\hline qs & 27,86 & 24,22 & 23,25 & 56,60 & 50,11 & 44,95 \\
\hline $\mathbf{E}$ & 1749,15 & 1420,39 & 1355,00 & 1153,36 & 1628,29 & 2721,04 \\
\hline $\mathbf{r}^{2}$ & 0,86 & 0,87 & 0,87 & 0,85 & 0,62 & 0,58 \\
\hline$\% \Delta \mathbf{E}$ & 57,66 & 57,58 & 57,23 & 80,57 & 47,02 & 78,86 \\
\hline
\end{tabular}




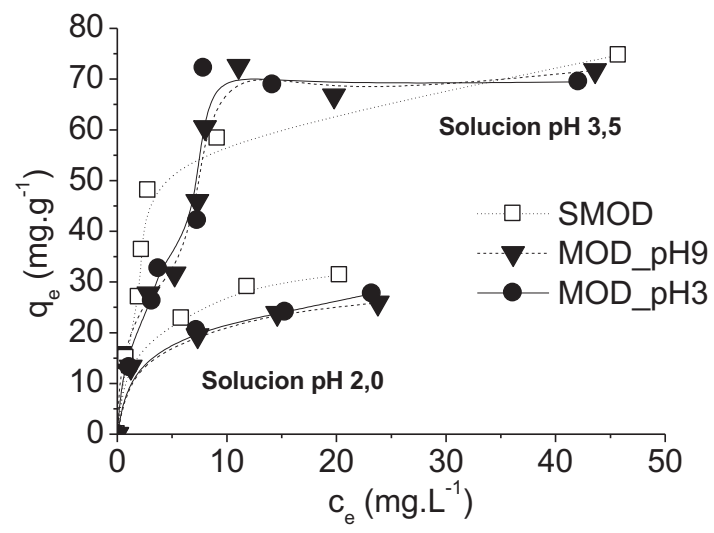

Figura 7. Isotermas de adsorción de los CA modificados y sin modificar en soluciones de distinto $\mathrm{pH}$.

Lu et al. ${ }^{21}$ reportaron que el mercurio presentó un mejor ajuste con el modelo de Langmuir. Arshadi et al. indicaron que el modelo de Langmuir-Freundlich mostró un mejor ajuste para la remoción de mercurio empleando partículas de cloruro de manganeso. Asasian et al. ${ }^{23}$ reportaron un mejor ajuste con el modelo de Freundlich para la remoción de mercurio.

\section{CONCLUSIONES}

1. El presente estudio muestra que el empleo de ultrasonido y una solución de nitrato de manganeso a pH 9 favorecen la formación de óxidos metálicos en la superficie del carbón activado.

2. Las cinéticas de adsorción fueron del tipo de pseudo segundo orden. Además, el mecanismo de adsorción predominantemente es del tipo Freundlich de acuerdo a las isotermas de adsorción.

3. Los carbones modificados con óxido de manganeso presentaron un aumento de su carga cero, siendo la adsorción de mercurio más efectiva pasando el punto de carga cero de dichos carbones. Los CA modificados presentaron un incremento en su punto de carga cero debido al incremento de los grupos fenólicos en su superficie.

4. La adsorción de mercurio fue mayor en el carbón activado modificado en comparación con el carbón activado sin modificar a un $\mathrm{pH}$ mayor al punto de su carga cero. El CA MOD_pH9 alcanzó una mayor capacidad de adsorción que MOD_pH3.

\section{AGRADECIMIENTOS}

Este trabajo fue financiado por la Dirección de Gestión de la Investigación de la PUCP, a través de la subvención DGI 2014-0051. 


\section{REFERENCIAS BIBLIOGRÁFICAS}

1. Arshadi M. Manganese chloride nanoparticles: A practical adsorbent for the sequestration of $\mathrm{Hg}$ (II) ions from aqueous solutio. Chem Eng J. 2015; 259: 170-182.

2. Mallyekkal S, Phillip L,. Pradeep T. As(III) removal from drinking water using manganese oxide coated alumina: performance evaluation and mechanistic details of surface binding. Chem Eng J. 2009; 153(1-3): 101-107.

3. Lisha K, Maliyekkal S, Pradeep T. Manganese dioxide nanowhiskers: A potential adsorbent for the removal of $\mathrm{Hg}$ (II) from water. Chem Eng J. 2010; 160(2): 432-439.

4. Henriques B, Goncalves G, Emami N, Pereira E, Vila M, Marques P. Optimization graphene oxide foam with enhanced performance and high selectivity for mercury removal from water. J Hazard Mater. 2016; 301: 453-461.

5. Obregón-Valencia D, Sun-Kou M. Comparative cadmium adsorption study on activated carbon preparaed from aguaje (Mauritia flexuosa) and olive fruit stones (Olea europea L.). J Environ Chem Eng. 2014; 2(4): 2280-2288.

6. Sun-Kou M, Obregón-Valencia D, Pinedo-Flores A, Paredes-Doig A, Aylas-Orejón J. Adsorption of heavy metals using activated carbons prepared from aguaje stones. Rev Soc Quím Perú. 2014; 80(4): 226-236.

7. Lalhmunsiama, Mok Lee S, Tiwari D. Manganese oxide immobilized activated carbons in the remediation of aqueous wastes contaminated with copper (II) and lead (II). Chem Eng J. 2013; 225: 128-137.

8. Pérez N, Sánchez M, Rincón G, Delgado L. Study of the behavior of metal adsorption in acid solutions on lignin using a comparison of different adsorption isotherms. Lat Am Appl Res. 2007; 37(2): 157-162.

9. Taffarel R, Rubio J. Removal of Mn2+ from aqueous solution by manganese oxide coated zeolite. Miner Eng. 2010; 23: 1131-1138.

10. Tiwari D, Laldanwngliana C, Choib C, Mok S. Manganese-modified natural sand in the remediation of aquatic environment contaminated with heavy metal toxic ions. Chem Eng J. 2011; 171(3): 958-966.

11. Deshetti J, Ippolito S, Sabri Y, Tardio J, Selvakannan PR, Nafady A, Reddy B, Bhargava S. Ceria-zirconia modified $\mathrm{MnOx}$ catalysts for gaseous elemental mercury oxidation and adsorption. Catal Sci Technol. 2016; 6: 1792-1803.

12. Xu H, Qu Z, Zong C, Huang W, Quan F. Yan N. MnOx/Graphene for the Catalytic Oxidation and Adsorption of Elemental Mercury. Environ Sci Technol. 2015; 49(11): 6823-6830.

13. Faulconer E, Mazyck D. Influence of activated carbon surface oxygen functionality on elemental mercury adsorption from aqueous solution. Environ Chem Eng. 2017; 5(3): 2879-2885.

14. Tang Q, Huang X, Chen Y, Liu T, Yang Y. Characterization and catalytic application of highly dispersed manganese oxides supported on activated carbon. J Mol Cat A-Chem. 2009; 301(1-2): 24-30.

15. Di Natale F, Erto A, Lancia A, Musmarra D. Mercury adsorption on granular activated carbon in aqueous solutions containing nitrates and Chlorides. J Hazard Mater. 2011; 192 (3): 1842-1850. 
16. Huang S, Ma C, Liao Y, Min C, Du P, Jian Y. Removal of mercury(II) from aqueous solutions by adsorption on poly(1-amino-5-chloroanthraquinone) nanofibrils: Equilibrium, kinetics, and mechanism studies. J Nanomater. 17 de marzo de 2016;2016:7245829. doi: 10.1155/2016/7245829

17. Awad F, El-Shall S. Effective removal of mercury(II) from aqueous solutions by chemically modified graphene oxide nanosheets. Arab J Chem. 2020, 13(1): 2659-2670.

18. Kadirvelu K, Kanmani P, Senthilkumar P, Subburam V. Separation of mercury(II) from aqueous solution by adsorption onto activated carbon prepared from Eichhornia crassipes. Sci Technol. 2004, 22(3): 207-222.

19. Tang J, Huang Y, Gong Y, Lyu H, Wang Q, Ma J. Preparation of a novel graphene oxide/ Fe-Mn composite and its applications for aqueous $\mathrm{Hg}(\mathrm{II})$ removal. J Hazard Mater. 2016; 316: 151-158.

20. Li Y, Xia M, An F, Ma N, Jiang X, Zhu S, et al. Superior removal of $\mathrm{Hg}(\mathrm{II})$ ions from wastewater using hierarchically porous, functionalized carbon. J Harzard Mater. 2019; 371(5): 33-41.

21. Lu X, Jiang J, Sun K, Wang J, Zhang Y. Influence of the pore structure and Surface chemical properties of activated carbon on the adsorption of mercury from aqueous solutions. Mar Pollut Bull. 2014; 78(1-2): 69-76.

22. Rao M, Reddy D, Venkateswarlu P, Seshaiah K. Removal of mercury from aqueous solution using activated carbon prepared from agricultural by product/waste. J Environ Manage. 2009; 90(1): 634-643.

23. Asasian N, Kaghazchi T, Soleimani M. Elimination of mercury by adsorption onto activated carbon prepared from the biomass material. J Ind Eng Chem. 2012; 18(1): 283-289. 